\title{
FIVE AXIOMS FOR POINT AND TRANSLATION IN AFFINE GEOMETRY
}

\author{
BY A. A. BENNETT
}

1. Introduction. Postulate systems for geometry are so numerous that the development of a new system is of itself of little interest. Before explaining the present system, some remarks about various lines of approach to geometry may be of value in estimating its significance.

(1) The introduction of a new form of geometry upon the basis of an assumed familiarity with other geometrical studies is a well known practice. This is what is done in the usual treatment of analytical geometry, of descriptive geometry, and frequently of projective geometry. There is even a tendency these days to teach euclidean geometry along semi-intuitional lines. This procedure appeals to elementary students as being "concrete", but it makes any appreciation of the logical structure of the subject difficult.

(2) A "synthetic" axiomatic treatment of geometric figures is the classical and still the orthodox line of approach. This is the method of Euclid, of the familiar non-euclidean studies, of Hilbert's Foundations, and of Veblen and Young's Projective Geometry, not to mention others. This is however subject to the disadvantage that the employment of powerful and economical analytical methods is necessarily delayed. It has also in the past been the innocent cover for much inaccurate reasoning. While a wide acquaintance with actual synthetic theorems is essential to the neat handling of complicated relations, it is difficult to justify an avoidance of analytical tools when these would simplify the discussion. The utility of quasi-analytical notions is admitted even by the extreme euclidean purists, not only in the simpler relations but when these appear under such titles as "method of similar figures", "method of translation", "methods of rotation and reflexion", "method of 
inversion". Of course algebraic methods can be proved justifiable on this basis, addition and multiplication being matters of definition, as Euclid did to some extent with his theory of proportion, and has been done completely by modern mathematicians. But there is at least a long, and to many students, painful delay, in the introduction of these algebraic operations.

(3) An axiomatic treatment based upon point and transformation may be followed, where the transformations are of the most general type leaving the propositions invariant. This is in keeping with Klein's famous Programm, is suggested by the non-axiomatic treatment of Lie, and has been carried out by Pieri, Hilbert, R. L. Moore, Gaba, and others. Here analytic machinery early suggests itself, but is not at once available. The transformation in its full generality impresses most students as neither an elementary nor an obvious notion, and so in a sense psychologically undesirable for a basic undefinable. The more familiar special transformations, such as translations, are found to be difficult of definition. Even in the case of such an elementary figure as a straight line, an extensive and intricate study is required before a definition is available.

(4) The number field may be first constructed, and geometry identified with the study of a hypercomplex algebra, addition and multiplication in the number field being developed in parallel. This is the characteristically analytical method, and that usual in vector analysis. Objections to the customary treatment are many. For instance there may be much duplication incident to a repetition for the hypercomplex numbers of the rules laid down for the original number field. Despite a possible formal independence, multiplication cannot be regarded as largely arbitrary. A thorough study of addition among points conditions in advance many of the properties that must be assumed for any useful system of multiplication, so that the extent to which multiplication may be defined in any given case is a matter of interest. Another frequent criticism of the 
hypercomplex treatment is due to its usual dependence upon coordinate systems, and the absence of any early test of purely geometrical, that is, of invariantive properties.

2. Proposed Conditions. It would seem not without interest to approach geometry on an axiomatic basis with the following conditions in mind.

(1) Analysis shall be available early.

(2) Save that congruence conditions are postponed, the system shall be essentially euclidean from the start.

(3) The analysis shall be of the simplest and most obvious sort, even at the possible sacrifice of symmetry.

(4) The one-dimensional case shall not be featured with special emphasis at the start.

(5) No special frame of reference shall be made fundamental.

A word as to these conditions. As to (1), no discussion should be required provided that it is not inconsistent with the retention of other ideals. As to (2), by postponing congruence conditions, all theorems become available not merely for the euclidean case but also for affine geometry, for which, as for projective geometry, there is no universal invariant for a point pair. Condition (3) gracefully excludes projective geometry with its extraneous factor of proportionality. Conditions (4) and (5) suggest that addition and multiplication be considered throughout as applied to hypercomplex numbers, and that further only their geometrically invariant features be discussed.

Beside the undefined element, point, it is natural from this line of attack to take as undefined the operation translation. Thus vector addition and subtraction may be expected to appear early in the discussion. The general notion of an affine transformation is eventually required. The question arises as to how far this may be secured by mere definition. From this stage on, the situation agrees in its essentials with that arising in general projective 
geometry. Within a single net of rationality no new undefined notion is required. For the general concept of straight line in its relation to affine transformations, a "fundamental" postulate is needed, and for the euclidean theory, not only must congruence be specialized, but order relations also must be established. One might refer for such questions to Veblen and Young's Projective Geometry.

3. The Five Axioms. The present brief study is confined for brevity and simplicity to the particularly elementary case of a finite geometry based on a Galois field where the prime modulus appears only to the first power, so that the field is modular in the primitive sense. It will be noted that three distinct "spaces" are considered, that of "points", that of "translations", and that of "automorphisms". The set of axioms is not categorical. Modifications are necessary in extension to cases which will admit the euclidean real or complex geometry as special instances, along the lines indicated above. Since the purpose of this set is to point out the extensive deductions inferrable from so few and apparently such mild conditions, and is not the study of this special and trivial type of space, questions of independence and completeness will not be discussed. The axioms together with some familiar and perhaps unnecessary definitions follow. "Point" and "translation" only are undefined.

Ax. I. $P$ is a finite set of elements.

The elements of $P$ may be called points and designated by the use of the letter $p$, with or without subscripts or other diacritical marks. By a permutation of $P$ is meant an operation based upon a biuniform correspondence among the elements of $P$, by which each element of $P$ is replaced by its uniquely corresponding element of $P$.

Ax. II 1. $A$ is a set of permutations of $P$.

The elements of $A$ may be called translations and designated by the use of the letter $a$, with or without subscripts or other diacritical marks. With respect to a given 
permutation, an element of $P$ is said to be fixed, if the permutation replaces this element by itself. The identical permutation is that which leaves each element fixed. We have not required that $A$ be a group or even a semi-group, but there will be a group of permutations of $P$ generated by the elements of $A$ and their inverses, or as we may say, by the set $A$.

Ax. II 2. The identical permutation is the only permutation in the group generated by the set $A$ which leaves as much as a single element of $P$ fixed.

Ax. II 3. For the given set, $P$, it is not possible to extend the set $A$, by adjoining further permutations, so that the extended set shall also satisfy II 1 , and II 2 .

From these axioms it follows readily that $A$ is itself a group. With a little discussion of the consequences of $A$ being possibly intransitive in $P$, it is seen that if $A$ were intransitive, a new permutation, $b$, could be adjoined, commutative with each $a$, and interchanging subsets of $P$ within each of which $A$ is transitive. Furthermore the set $A$ together with $b$ would satisfy II 1 and II 2 , by making explicit use of the commutative character of $b$, so that II 3 would fail to hold if $A$ were not transitive. Zermelo's axiom in the finite case is assumed as a principle of logic.

For an unknown set $P$, the axioms II place no restrictions upon $A$ other than that $A$ shall be a group. For a given group $A$, the set $P$ is found to be in biuniform correspondence with $A$ and in as many ways as one chooses an element of $P$ to correspond to the identical permutation. No points of $P$ are specialized, while in $A$ there is at least one element not to be confused with any other, namely, the identical permutation. Two permutations, which are elements of $A$, are said to be equivalent, if and only if there is an automorphism of $A$ (hence preserving the multiplication table of $A$ ), which replaces the first by the second, and also an automorphism of $A$ replacing the second 
by the first. Since an automorphism may replace distinct elements by a single one, this double condition is needed.

Ax. III. Any two elements of A, neither of which is the identical element, are equivalent.

We may now conclude that with the exception of the identical element, all elements of $A$ are of the same order. Since any cyclic group of composite order has proper cyclic subgroups, we conclude that the common order of all nonidentical elements is a prime, say $p$. The total order of the group can contain no prime factor other than this $p$, so that since the set is of finite order, this total order may be designated by $p^{n}$. We may call this $n$ the dimensionality of $P$ and of $A$. Any set of $p$ points, each of which is obtainable from each other by the operations of a cyclic subgroup of $A$, may be called a line. Thus each line has $p$ distinct points.

It is a well known theorem* in the study of finite groups that every prime power group contains elements other than the identity commutative with every other element of the group. Applying this to $A$, and using the fact that the elements, $a$, other than the identity are equivalent, we infer that $A$ is commutative. We may now identify the operation of obtaining the result of two successive permutations, with the operation of adding these elements, $a$.

The totality of automorphisms of $A$ is called the set $M$. Thus if $m$ is an element of $M$, and $a_{1}$ and $a_{2}$ are two elements of $A$, it follows that $m\left(a_{1}+a_{2}\right)=m\left(a_{1}\right)+m\left(a_{2}\right)$. Given two automorphisms, $m_{1}$ and $m_{2}$, and denoting by $a$ an unrestricted variable in $A$, we may define $a^{\prime}$ and $a^{\prime \prime}$ by the conditions $m_{1}(a)=a^{\prime}, m_{2}(a)=a^{\prime \prime}$. Now there is an automorphism replacing $a$ by the sum $a^{\prime}+a^{\prime \prime}$, since there is obviously such a uniform correspondence and it satisfies the distributive condition essential for an auto-

* Cf. Hilton, Finite Groups, p. 142. 
morphism, since in fact $\left(a_{1}^{\prime}+a_{2}^{\prime}\right)+\left(a_{1}^{\prime \prime}+a_{2}^{\prime \prime}\right)=\left(a_{1}^{\prime}+a_{1}^{\prime \prime}\right)$ $+\left(a_{2}^{\prime}+a_{2}^{\prime \prime}\right)$. Since $M$ contains each automorphism of $A$, it contains this also. We shall naturally designate it by $m_{1}+m_{2}$. Thus $M$ is completely distributive with respect to the addition of elements of $A$.

The result of the successive application of two elements of $M$ is again in $M$, and $M$ is found to constitute a semigroup, which furthermore contains an identical element for the semigroup. The terms multiplication and product may be used in a strict sense for this $M$. While $M$ is a semigroup with respect to multiplication, it may be shown to form with respect to addition, as already used, a group. Its identical element with respect to addition is the nullautomorphism, that which replaces each element of $A$ by $O$, the identical element of $A$. The element of $M$ identical with respect to multiplication is of course that which replaces each $a$ by itself. Thus $M$ taken with its rules of operation constitutes a linear algebra. This $M$ includes singular automorphisms, that is automorphisms replacing distinct elements of $A$ by a common element. It is therefore no group. Whether approached from the side of the modular theory of numbers, or from the side of specializations in the theory of general projective geometry, it is found that $M$ is simply isomorphic with the semigroup of all affine projectivities among translations in a modular space, modulo $p$, of $n$ dimensions, leaving the null translation invariant, and with the semigroup of all linear homogeneous substitutions in $n$ variables, taken modulo $p$, no factor of proportionality being suppressed.

In the one-dimensional case, multiplication is commutative in $M$ and the number of elements in $M$ is the same as in $P$ and $A$. For cases of higher dimensionality, there are $p^{n^{2}}$ elements in $M$ and multiplication is not commutative, although within any cycle multiplication is of course always commutative.

The University of Texas 